\title{
From "Hooah" to "Om": Mindfulness Practices for a Military Population
}

\author{
Kimberlee B. Bonura, $\mathrm{PhD}$ \\ Walden University, Minneapolis, Minnesota, United States \\ (iD) https://orcid.org/0000-0003-4273-2800 \\ Dawn M. Fountain, $\mathrm{PhD}$ \\ University of West Florida, Pensacola, Florida, United States \\ Contact: kimberlee.bonura@mail.waldenu.edu
}

\section{Abstract}

Mindfulness practices, in general, have a growing body of evidence of effectiveness for improving both physical health and mental health. Further, these practices are showing promise when implemented with military populations for mental health issues such as posttraumatic stress disorder. Challenges arise for practitioners in understanding the military's cultural differences and the functional aspects of mindfulness that may be specifically useful to military members. We outline strategies for referring military members to quality services and instruction, as well as best practices for connecting with military clients. Recommendations are made for further research with female service members and using single-case design.

Keywords: mindfulness, military, PTSD, yoga, meditation

Date Submitted: May 10, 2020 | Date Published: June 30, 2020

\section{Recommended Citation}

Bonura, K. B., \& Fountain, D. M. (2020). From "hooah" to "om": Mindfulness practices for a military population. Journal of Social, Behavioral, and Health Sciences, 14, 183-194. https://doi.org/10.5590/JSBHS.2020.14.1.13

\section{Introduction}

Mind-body medicine are practices that focus on learning control of the mind to improve the health and wellness of the body. These include mindfulness interventions, such as meditation, mindfulness-based stress reduction (MBSR), yoga, and martial arts. Mindfulness is a quiet awareness of one's internal and external world using the five senses while engaging in any activity (Schiraldi, 2009). An understanding of intentional mindfulness is the first step in mindfulness interventions, whether it is relaxation breathing with an internal focus or purposeful movement patterns of yoga with an interaction of internal and external (Weiler, 2013). There is a growing body of empirical evidence demonstrating the effectiveness of mind-body strategies for improving psychological health, reducing depression and anxiety, improving stress resilience and coping

Note: On behalf of all authors, the corresponding author states that there is no conflict of interest. This research was not funded. This article was a research review and does not contain any studies with human participants or animals performed by any of the authors. 
skills, and facilitating self-care and physical health through stress reduction benefits (Grossman et al., 2004). Mind-body strategies have been found to reduce blood pressure (Palta et al., 2012), reduce cholesterol (Ornish et al., 1998), and reduce pain symptoms (Zeiden et al., 2011).

Mindfulness practices such as yoga, meditation, and Tai Chi offer the potential to support military populations (including active duty, veterans, and military families) as both an adjunct to therapy and as a preventative stress-management technique (Gray \& Litz, 2005; Lombardo \& Gray, 2005). Mindfulness practices may support recovery for individuals with posttraumatic stress disorder (PTSD; Bormann et al., 2008; Schiraldi, 2009; Stankovic, 2011; Vujanovic et al., 2011). Likewise, mindfulness practices may help with pain management in veterans (Groessl et al., 2008). Mindfulness practices can also help soldiers cope with predeployment stress (Stanley et al., 2011), decrease sleep disturbances (Harb et al., 2009), and support the development of soldier resilience (Rees, 2011). Mindfulness practices may also support military care providers to prevent burnout and compassion fatigue in those who provide support services to soldiers (Duerr, 2008; Welcome Home Vets, Inc., 2011). One survey of active duty military, retirees and spouses reported that $81 \%$ of respondents had used complementary and alternative medicine (CAM) strategies (including mindfulness techniques) as adjuncts to health care; most indicated that they would like CAM treatments to be available at military treatment facilities (McPherson \& Schwenka, 2004).

Military populations may have unique challenges related to the use of mindfulness practices. Individuals in the military may have misconceptions of mindfulness practices and feel that mindfulness practices are contradictory with the "warrior ethos" (Cantrell \& Dean, 2007; Sheppard et al., 2010; Tick, 2005). Likewise, access issues may occur for individuals stationed in remote areas. Further, mindfulness instructors may have limited experiences with a military population and minimal understanding of the unique stressors of military lifestyle (Sheppard et al., 2010).

The purposes of this article are to (a) review the benefits of mindfulness practices for a military population and (2) provide guidelines for using mindfulness practices with active duty military, veterans, and military families. While the article is primarily informed by research and information about the U.S. military forces, implications and recommendations may be relevant for other military populations; specific national and cultural contexts should be considered for non-U.S. military populations. The article will support mindfulness practitioners who want to ensure quality of service in teaching mindfulness strategies to military populations. The article will also support mental health providers and health care providers who want to understand appropriate applications of mindfulness techniques as an adjunct to mental health care for military populations and to offer strategies for identifying qualified mindfulness practitioners for referrals and collaborative work in support of the mental health care of active duty military, veterans, and military families.

\section{Defining Military Populations}

Bonura and Lovald (2015) have proposed that effective support of military members requires the development of "military cultural competency." Practitioners should have a baseline understanding of military populations, including the unique needs, challenges, and opportunities for service to population subgroups (i.e., active duty service members, prior service members, including disabled veterans and retirees, and military family members). Military clients bring with them a unique set of experiences, insights, challenges, and skills. Each one of them has been exposed to and influenced by their branch of service, rank structure, and military operations (Sheppard et al., 2010). Developing a plan to do this effectively may present a unique challenge for a predominantly civilian health care environment (Sheppard et al., 2010). 
Bonura \& Fountain, 2020

\section{Military Population Demographics}

Only 0.5\% of the American population serves in the armed forces at any given time (Eikenberry \& Kennedy, 2013). According to the 2019 Bureau of Labor Statistics report, only 8\% of adults in the United States are veterans. As of 2018, women are $17 \%$ of the active duty force (Kamarck, 2019). Women make up 10\% of the total population of veterans (Bureau of Labor Statistics, 2019). Among adult women in the United States over the age of 18 , only $2 \%$ have prior military service (Newport 2012). Twenty-four percent of adult men are veterans, but only $12 \%$ of men aged $25-34$ are veterans, and only $14 \%$ of men aged $35-39$ are veterans. The higher overall percentage of men with prior military status reflects the larger proportion of older men who served via the draft during prior conflicts such as World War II, Korea, and Vietnam. Among men over age 65, more than 50\% have prior military service, and among men aged $85-89,80 \%$ have prior military service. Significantly, though the veteran population as a portion of the overall U.S. population has been decreasing since 1990 (as older male veterans have died), the percentage of the veteran population who have a serviceconnected disability has continually increased since 1990 (U.S. Department of Veterans Affairs, 2019a). As of 2017, according to the Department of Veterans Affairs (2017a), the largest population of veterans served in the Vietnam War (more than 7 million veterans still living). However, among those veterans receiving disability compensation, there are more veterans receiving disability benefits related to the Persian Gulf Wars (including all U.S. involvement in the Middle East since 1990) than there are living veterans receiving disability compensation for all prior conflicts (U.S. Department of Veterans Affairs, 2017a).

It is helpful for health and wellness providers and individuals within health care settings who serve military populations to have some understanding of the military, in order to serve these individuals (Sheppard et al., 2010). It is also helpful to understand the differences in experience that veterans may have, based on their era and time of service, and their level of disability. Practitioners should consider that among younger veterans, regardless of disability level, experience in a combat zone is likely. A 2011 RAND report indicated that $73 \%$ of active duty Army members had deployed to either Iraq or Afghanistan (Baiocchi, 2013). While the integration of women into the combat arms (i.e., infantry and armor branches in the Army) has been occurring slowly since 2016 (Kamarck, 2019), many female service members have already and continue to deploy to combat zones in military specialties such as intelligence, foreign language, and medical. Specific statistics regarding the percentage of female service members with at least one deployment to Iraq and Afghanistan could not be identified. Research indicates that female service members may experience unique psychological stressors in response to combat zone deployments (Gibbons et al., 2012).

\section{The Military Lived Experience}

There are key components to military structure which may impact how the military individual engages. It is helpful for both leaders at the institutional level and practitioners at the individual level to understand the general military structure and consider the specific demographics and requirements of the military clients in their setting or institution. The Army, Air Force, Navy, Coast Guard, and Marines each have a unique culture and an individual affiliated with each of these branches of service will likely reflect the culture and mission of their branch of service. Furthermore, rank structure affects personal identity within the service, with different norms and standards for enlisted, noncommission officers (i.e., senior enlisted) and officer ranks (Bonura \& Lovald, 2015; Sheppard et al., 2010).

There are differences in the lived military experience for active duty service members versus those in the reserves. Likewise, reserve service members may experience differences based on reserve status, particularly reserve service members on permanent active duty or called to active duty during war. Further, cultural differences based on military structure will affect all of those within the culture, and family members may experience and reflect those differences. The experiences of an Army spouse may be fundamentally different from the experiences of a Marine Corps spouse, just as the experiences of the spouse of an enlisted service 
Bonura \& Fountain, 2020

member will be distinct from those of an officer spouse. Family members of active versus reserve service members will likewise have unique challenges. Within the spouse population, civilian female spouses of military service members will experience different challenges than will civilian male spouses of military service members, as will service members who are married to another service member (Bonura \& Lovald, 2015; Sheppard et al., 2010).

When working with military individuals, consider each person's history of military training, acquisition of reflexive skills, and history of reinforcement. These are part of military cultural norms. A service member's military occupation specialty has a set of relevant trained skills. Consider the differences in training between someone trained in information technology related to electronic warfare and those trained in hand to hand combat such as members of the infantry. There may be significant differences between training focused on computer interaction with an enemy versus learning how to interact with the enemy in hand-to-hand combat. Overarching all of the military occupation specialty trainings is the fight like you train mentality. Even in simulations, the service member is expected to be operating at $100 \%$ capacity. The trained skills become second nature and unintentional, even reflexive. A keen observation ability and focus on all external stimuli are hallmarks of these skills. The strong ability to evaluate external factors like topography and enemy positions saves lives. Situational awareness and high levels of vigilance are rewarded through successful missions but also from the command leadership. Promotions and commendations are given to those who excel in managing the external world. The extensive training, reflexive skills, and the support that comes from this external focus may be the opposite of an internal mindfulness-based focus, even if both require present moment awareness. The internal awareness of oneself required in mindfulness practices may feel counterintuitive in contrast to the basic training of a service member. Simply expressed, not paying attention to what is going on in the environment may be considered a dereliction of duty (Cantrell \& Dean, 2007; Sheppard et al., 2010; Tick, 2005).

\section{Defining a Prevalent Psychological Disorder in the Military Population}

The fifth edition of the Diagnostic and Statistical Manual of Mental Disorders (American Psychiatric Association, 2013) provides a category for Trauma and Stressor-Related Disorders. Military service members are a cross section of society and may experience any type of clinical mental disorder in their life. The prevalence of PTSD among veterans of military combat is from 30-50\% (American Psychiatric Association, 2013). Diagnosis includes exposure to a traumatic event, intrusive symptoms, avoidance behaviors, negative cognitions and mood, high levels of arousal, and clinical distress in social and daily functioning. There can be a delayed expression of symptoms, even years after the trauma. The Diagnostic and Statistical Manual of Mental Disorders (fifth edition) explains that military service members' prognosis may be affected by environmental factors such as whether they participated in war behaviors, such as killing a combatant, or viewing others engaging in war behaviors. PTSD is associated with an increased risk of suicide. Traumatic brain injury may exist as a different diagnosis as it is a common physical injury for those experiencing combat. Neurocognitive symptoms may overlap between PTSD and traumatic brain injury, as there is a $48 \%$ likelihood of co-occurrence (American Psychiatric Association, 2013). People with PTSD have an 80\% increase in other mental disorders, depression, bipolar, or anxiety (American Psychiatric Association, 2013). Military members' substance use has been estimated up to 40\% (Hoge et al., 2004; Jakupcak et al., 2010).

It is also helpful to understand that all military conflicts, past and present, are an attack on the senses (Hoge et al., 2004). This may have implications for mindfulness and internal awareness training. Although not specifically exposure training, initial experiences with mindfulness training may lead to remembering conflict experiences and cause distress (Gray \& Litz, 2005). Practitioners should be prepared for this before 
Bonura \& Fountain, 2020

implementing mindfulness strategies and provide the opportunity for support and debriefing (Gray \& Litz, 2005; Turner et al., 2005).

\section{When Mindfulness Works to Support Psychological Health}

Research with veterans has found that those with PTSD are about 25\% more likely than veterans without PTSD to use CAM treatments for stress management (Micek et al., 2007). A 2011 survey of all Veterans Administration hospitals found that $89 \%$ of Veterans Affairs (VA) facilities offered some form of CAM treatment, primarily in the treatment of stress management, anxiety disorders, PTSD, depression, and pain (Veterans Affairs Healthcare Analysis and Information Group, 2011). These statistics speak to a growing understanding that while conventional medicine is effective when dealing with acute trauma and illness, military populations need more support and care with chronic issues and conditions exacerbated by stress. To further understand this relationship, Cushing and Braun (2018) conducted a meta-analysis of the use of mind-body therapies, including yoga and mindfulness, for PTSD in veterans; across 15 included studies, results indicated that mind-body therapies offer promise for supporting veterans in managing PTSD symptoms. At present, VA does not recommend complementary strategies as a primary treatment approach for PTSD; in accordance with VA best practices, many VA facilities use complementary strategies, such as mindfulness-based training, as a complement to therapy and pharmacological interventions (U.S. Department of Veterans Affairs, 2017b).

Different approaches may be necessary for different military populations. Practitioners should consider their ability to work with the various groups, including active duty military; disabled former military or medically retired military; retired military, who may or may not have service-connected disabilities and health issues; and military spouses and children (Dekel \& Monson, 20102; Lombardo \& Gray, 2005; Turner et al., 2005). General considerations when working with the military are to have a basic understanding of the structure as discussed (i.e., the five primary branches of service, officer versus enlisted, active duty versus reserves), and to understand that the basic organizational and leadership structure is layered and hierarchical, from the bottom up. Even the lowest ranks, young adults who may be recently out of high school, have a great deal of responsibility and autonomy in their position (Sheppard et al., 2010).

A commonality among many treatments for PTSD and associated disorders is that they are multicomponent and are usually implemented stepwise through phases (Foa et al., 2009). Several protocols include relaxation training and may also include breathing techniques, meditation, muscle relaxation, and movement modalities such as yoga. From an experimental perspective, when multicomponent interventions do not include component analysis, it is difficult to determine which aspects are effective (Foa et al., 2009). Still, multicomponent strategies are considered to be the best approaches for PTSD and trauma-related stress (Gray \& Litz, 2005; Turner et al., 2005).

There are several other treatment protocols with demonstrated potential for combat-related PTSD symptoms, for instance, cognitive processing therapy, dialectical behavior therapy, group therapies, art therapy, and psychopharmacotherapy. While these methodologies are not primarily mindfulness-based, they may include mindfulness strategies in their implementation (Foa et al., 2009).

\section{Relaxation and Deep Breathing}

Broadly classed as stress management techniques, slow, diaphragmatic breathing has been used to decrease physiological arousal and negative mood. Progressive muscle relaxation addresses physical tension (Foa et al., 2009). Both require a practice of mindfulness. Research consistently shows that mindfulness training is an effective strategy in helping people learn how to manage stress and reduce anxiety. Deep breathing is 
particularly effective for reducing anxiety. Learning to control the breath is a powerful strategy, because it helps the individual learn to control energy, awareness of anxiety, and reactivity to stressors. Deep breathing also conditions the autonomic nervous system to be less reactive overall. One study found that when participants practice yoga breathing strategies for 25 minutes per day over six months, their anxiety scores dropped by 44\% (Doria et al., 2015).

\section{Best Practices for Mindfulness with a Military Population}

Both authors are experienced yoga instructors and practitioners with experience teaching military populations. Collectively, the authors have gained approximately 50 years of experience as members of military families, as well as through working on military bases, and receiving extensive military support training. Best practices offered in this section reflect the authors' experiences teaching mindfulness strategies to military populations and in military settings, along with empirical evidence in the literature regarding best practices for interaction with military populations. As practitioners, it is essential to take advantage of any continuing education opportunities provided by the VA or veteran's organizations like Welcome Home Vets. These opportunities will likely offer additional information on best practices working with vets by instructors who are vets (Bonura \& Fountain, 2014; Kognito, 2018).

\section{Identifying Qualified Mindfulness Instructors}

Practitioners who intend to recommend mindfulness strategies to military individuals as a supportive strategy for stress management, should first gain familiarity with mindfulness practices by trying various practices. For instance, mental health providers and health care providers may want to try meditation, yoga, MBSR, Tai $\mathrm{Chi}$, and other martial arts. It may also be helpful to access academic journals publishing empirical research on mindfulness practices, including work specifically within the military setting or at the Veteran's Affairs hospitals. These articles provide a growing body of empirical evidence for the beneficial use of mindfulness strategies with military populations. It is also helpful for health care providers to establish relationships with appropriately qualified instructors in their areas. Look for a skilled, trained instructor with credentials through an appropriate, relevant credentialing organization, such as the Yoga Alliance (https://www.yogaalliance.org/) or the American Council on Exercise (https://www.acefitness.org). The individual should have a background in working with military population and understanding of unique challenges of military and be CPR certified if teaching a physical modality such as yoga, Tai Chi, or martial arts (Bonura \& Fountain, 2014).

\section{Recommendations in Practice}

Military clients are adults and usually very self-reliant; even children who are military family members may have higher levels of independent activity and responsibility than children in civilian families, due to the unique constraints of military life. Practitioners should avoid providing any value-laden comments or asking questions when someone leaves early or does not follow along in the mindfulness exercise. Military members appreciate standard operating procedures and structure. Best practices include written class schedules and staying on topic. Practitioners should be on time and fully prepared. Military members are used to operations not going as planned but appreciate when they do. In military lingo a change to a previous order is called a fragmentary order. Changing class structure can be like a fragmentary order. Practitioners should provide a no-chaos zone (Bonura \& Fountain, 2014).

\section{Family influence}

In working with military populations, practitioners should consider the stressors, needs, and influence of the military family members. For instance, military family members experience chronic stressors that are 
different from nonmilitary populations, including frequent moves and recurrent periods of separation from the military service member. According to the 2019 Blue Star Family Survey, more than a third of military families have no one in their local area to ask for a favor, such as assistance with childcare or transportation. These practical stressors may be essential to address in any stress-management training for military family members. Mindfulness-based training groups may offer an opportunity to form community connections for military family members. Practitioners should also consider how family members may influence the service member. Military spouses who practice mindfulness-based strategies may support the military service member in using mindfulness-based strategies as stress reduction, even if the military service member is not directly receiving mindfulness-based training.

\section{Language use}

In yoga, martial arts, and other Eastern-based disciplines, use basic routines, simple verbiage, and common names for positions. For instance, in yoga, use the phrase "warrior pose" instead of the Sanskrit name virabhadrasana (Bonura \& Fountain 2014; Weiler, 2013). Experienced yoga practitioners may find tools such as the Yoga Journal Pose Finder helpful in translating Sanskrit pose names to more user-friendly Englishbased descriptions and names (available at https://www.yogajournal.com/pose-finder).

\section{Modalities}

Consider different schools of yoga; some geared toward fitness yoga or gentle yoga may accommodate different student needs. For example, one derivative of hatha yoga is power yoga, a fast-paced format that includes aerobic activity. This more physically challenging yoga has found a home in many VA centers and military bases (Connected Warriors, http://connectedwarriors.org/). Older adult veterans within the VA hospital system may prefer adapted modalities, such as Chair-based Yoga, depending on their physical health and chronic pain.

\section{Focus on fitness}

Any reference to fitness and or physical training will make relaxation training more palatable. Consider deep breathing and progressive muscle relaxation as foundational strategies to support learning mindfulness techniques. Avoid more esoteric environmental approaches to mindfulness, for instance, do not use candles, incense, or mantras (Weiler, 2013).

\section{Practice sensitivity to each student}

Considering the hypersensitivity in some people with PTSD or general anxiety, instructors should limit handson corrective feedback and avoid close proximity to relaxation students. Additionally, instructors can suggest that eyes do not have to be closed for any of the activities. Keeping smaller class sizes, particularly in small classrooms, may reduce anxiety. Above all, teach to the needs of the student (Angeletti, 2016; Kognito, 2018; Weiler, 2013).

\section{Offer military analogies}

Relaxation and mindfulness strategies which are referred to in military history may support some military students in conceptualizing the practice. Effective recommendations for reading for both service members and practitioners include Sun Tzu's (ca. 500 B.C.) The Art of War, Tick's (2005) exploration of Native American warriors, and Homer's (ca. 800 B.C.) The Odyssey.

Sun Tzu's work is a mainstay in many military training schools. It is a treatise on leadership, control, intentional observation, and preparation before, during, and after conflict. Above all, the text expresses strategizing for success without battle through awareness of oneself and the environment. 
Tick (2005) expounded on Native American traditions, rituals, and healing the warrior's soul. The author recounts that returning warriors have acquired strength and wisdom. The veterans are honored and returned to normalcy by connecting with elders and completing rituals.

The ancient tale of Homer's Odyssey is the story of a returning veteran from the Trojan Wars. The detailed account describes Odysseus having flashbacks, avoiding family, experiencing guilt for having survived, and many other characteristics of PTSD. The allegory reflects the phases of returning to normal after war and shows treatment through support by family and comrades.

\section{Summary and Conclusions}

Overall, mindfulness-based modalities, including MBSR, yoga, Tai Chi, other forms of the martial arts meditation, and breathing training, offer potential to support military personnel in managing stress as well as clinical conditions. Mental health providers, educators, and mindfulness instructors should be informed about the various conditions for which mindfulness strategies are empirically supported and understand the unique needs and constraints of military service members, military veterans, and military family members (Fletcher et al., 2017). The VA already offers mindfulness-based interventions and supports the use of mindfulnessbased training with veterans, through their Whole Health for Whole Life program (U.S. Department of Veterans Affairs, 2019b). Mental health providers interested in using mindfulness-based strategies as an adjunct to care are encouraged to develop their own mindfulness-based practices to support understanding of both the benefits of and challenges inherent to mindfulness-based practices.

\section{Limitations}

Military service members are a cross-section of American society. It is important not to overgeneralize the military culture and characteristics as absolute. Military members are influenced by their premilitary experiences. Not all clinical characteristics present the same in every person. As such, to overgeneralize mindfulness as a panacea would be short-sighted. Further, recent critics are concerned with both the "McMindfulness" approach that strips mindfulness-based practices from their Buddhist context (Purser, 2019) and the potential religious complications of mindfulness for Western Christian individuals (Wong, 2018). Any discussion of mindfulness as a potential intervention must consider the cultural context and the personal needs and comfort of individual practitioners.

\section{Future Directions}

Even with the growing evidence, more research is needed on using mindfulness strategies with the military population as a possible treatment variable for PTSD, stress management, depression, and resilience training. Further, there is a need for more research with female service members. As Vietnam-era veterans are currently the largest population of veterans in the United States, research addressing the use of mindfulness as a strategy for managing pain and psychological distress among older adults may also be useful. Future research should combine both randomized controlled studies and single-case design.

Mindfulness interventions are not one size fits all. Individuals need individualized programs. Practitioners wishing to add to the body of evidence supporting mindfulness practice may implement the applied research method, single-case design (Bailey \& Burch, 2002). Single-case design is empirically supported and may be a more manageable approach for practitioners, thus allowing them to collect and present experimental data from successful programming for individuals (Bailey \& Burch, 2002; Müller-Engelmann et al., 2019). Although making an argument for applied single-case design is a topic for another article, it holds that practitioners already extrapolate successful intervention strategies for clinical use from published works 
regardless of methodology. A review by Cushing and Braun (2018) indicated successful outcomes of mindfulness modalities from both single group and randomized research.

Randomized controlled studies of mindfulness as an intervention for specific health conditions relevant to veteran populations may be feasible for grant-funded research at the institutional level within VA health care settings and may offer further support for medical funding for mindfulness health care interventions.

Individuals in the service of the nation have unique considerations in terms of clinical conditions and treatment protocols. The goals of this article were to disseminate useful information about mindfulness practices with military populations and to support improved clinical outcomes through an understanding of military culture and needs.

\section{References}

American Psychiatric Association. (2013). Diagnostic and statistical manual of mental disorders (5th ed.).

Angeletti, M. (2016). Teaching a trauma-informed yoga class. https://www.yogaalliance.org/Learn/Article_Archive/Teaching_a_Trauma-Informed_Yoga_Class

Bailey, J. S., \& Burch, M. R. (2002). Research methods in applied behavior analysis. Sage Publications.

Baiocchi, D. (2013). Measuring Army deployments to Iraq and Afghanistan. RAND Corporation. https://www.rand.org/pubs/research_reports/RR145.html

Blue Star Families. (2019). 2019 Military Family Lifestyle Survey. https://bluestarfam.org/survey/

Bonura, K. B., \& Fountain, D. (2014). From Hooah to Om: Mindfulness practices for a Military population. Poster at the 2014 Annual Conference of the American Psychological Association, Washington, D.C.

Bonura, K., \& Lovald, K. (2015). Military cultural competency: Understanding how to serve those who serve. Higher Learning Research Communications, 5(2), 4-13. http://journals.sfu.ca/liu/index.php/HLRC/issue/view/20

Bormann J., Thorp S., Wetherell J., \& Golshan S. (2008). A spiritually based group intervention for combat veterans with posttraumatic stress disorder: Feasibility study. Journal of Holistic Nursing, 2, 109116.

Bureau of Labor Statistics. (2019). Employment Situation of Veterans-2019. https://www.bls.gov/news.release/pdf/vet.pdf

Cantrell, B., \& Dean, C. (2007). Once a warrior: Wired for life. WordSmith.

Cushing, R. \& Braun, K. (2018). Mind-body therapy for military veterans with post-traumatic stress disorder: A systematic review. The Journal of Alternative and Complementary Medicine, 24(2), 106-114. https://doi.org/10.1089/acm.2017.0176

Dekel, R., \& Monson, C. (2010). Military-related posttraumatic stress disorder and family relations: Current knowledge and future directions. Aggression and Violent Behavior, 15, 303-309. https://doi.org/10.1016/j.avb.2010.03.001

Doria, S., de Vuono, A., Sanlorenzo, R., Irtelli, F., \& Mencacci, C. (2015). Anti-anxiety efficacy of Sudarshan Kriya Yoga in general anxiety disorder: A multicomponent, yoga based, breath intervention program for patients suffering from generalized anxiety disorder with or without comorbidities. Journal of Affective Disorders, 184, 310-317. https://doi.org/10.1016/j.jad.2015.06.011 
Bonura \& Fountain, 2020

Duerr, M. (2008). The use of meditation and mindfulness practices to support military care providers: A prospectus. Center for Contemplative Mind in Society.

http://www.contemplativemind.org/admin/wp-

content/uploads/2012/o9/MeditationforCareProviders.pdf

Eikenberry, K., \& Kennedy, D. (2013, May 26). Americans and their military, drifting apart. The New York Times. https://www.nytimes.com/2013/05/27/opinion/americans-and-their-military-driftingapart.html

Fletcher, C. E., Mitchinson, A. R., Trumble, E., Hinshaw, D. B., \& Dusek, J. A. (2017). Providers' and administrators' perceptions of complementary and integrative health practices across the Veterans Health Administration. Journal of Alternative \& Complementary Medicine, 23(1), 26-34. https://doi-org.ezproxy.lib.uwf.edu/10.1089/acm.2016.0236

Foa, E., Keane, T., Friedman, M., \& Cohen, J. (2009). Effective treatments for PTSD (2nd ed.). Guilford Press.

Gibbons, S., Hickling, E., Barnett, S., Herbig-Wall, P., \& Watts, D. (2012). Gender Differences in Response to Deployment Among Military Healthcare Providers in Afghanistan and Iraq. Journal of Women's Health, 21(5), 496-504.

Gray, M., \& Litz, B. (2005). Behavioral interventions for recent trauma. Behavior Modification, 29(1), 189215. https://doi.org/10.1177/0145445504270884

Groessl, E., Weingart, K., Aschbacher, K., Pada, L., \& Baxi. S. (2008). Yoga for veterans with chronic low-back pain. The Journal of Alternative and Complementary Medicine, 14(9), 1123-1129.

Grossman, P., Niemann, L., Schmidt, S., \& Walach, H. (2004). Mindfulness-based stress reduction and health benefits. Journal of Psychosomatic Research, 57, 35-43.

https://www.jpsychores.com/article/So022-3999(03)00573-7/abstract

Harb, G., Cook, J., Gehrman, P., Gamble, G., \& Ross, R. (2009). Posttraumatic stress disorder nightmares and sleep disturbances in Iraq war veterans: A feasible and promising treatment combination. Journal of Aggression, Maltreatment, \& Trauma, 18, 516-531. https://doi.org/10.1080/10926770903035150

Hoge, C., Castro, C., Messer, S., McGurk, D., Cotting, D., \& Koffman, R. (2004). Combat duty in Iraq and Afghanistan, mental health problems, and barriers to care. The New England Journal of Medicine, 351(1), 13-22.

Jakupcak, M., Tull M., McDermott, M., Kaysen, D., Hunt, S., \& Simpson, T. (2010). PTSD symptom clusters in relationship to alcohol misuse among Iraq and Afghanistan war veterans seeking post-deployment VA health care. Addictive Behaviors 35, 840-843. https://doi.org/10.1016/j.addbeh.2010.03.023

Kamarck, K. (2019). Diversity, inclusion, and equal opportunity in the armed services: Background and issues for Congress. https://fas.org/sgp/crs/natsec/R44321.pdf.

Kognito. (2018). Veterans on campus for faculty and staff. https://kognito.com/products/veterans-oncampus-for-faculty-staff

Lombardo, T., \& Gray, M. (2005). Beyond exposure for posttraumatic stress disorder (PTSD) symptoms. Behavior Modification, 29(1), 3-9. https://doi.org/10.1177/0145445504270853

McPherson, F. (2004). Use of complementary and alternative therapies among active duty soldiers, military retirees, and family members at a military hospital. Military Medicine, 169(5), 354-357.

Micek, M. A., Bradley, K. A., Braddock, C. H., et al. (2007). Complementary and alternative medicine use among Veterans Affairs outpatients. Journal of Alternative and Complementary Medicine, 13, 190193. https://doi.org/10.1089/acm.2006.6147 
Müller-Engelmann, M., Schreiber, C., Kümmerle, S., Heidenreich, T., Stangier, U., \& Steil, R. (2019). A trauma-adapted mindfulness and loving-kindness intervention for patients with PTSD after interpersonal violence: A multiple-baseline study. Mindfulness, 10(6), 1105. http://search.ebscohost.com/login.aspx?direct=true\&db=edb\&AN=136443541\&site=eds-live

Newport, F. (2012). In U.S., 24\% of men, $2 \%$ of women are veterans. Gallup. https://news.gallup.com/poll/158729/men-women-veterans.aspx

Ornish, D., Scherwitz, L., Billings, J., Gould, L., Merritt, T., Sparler, S., \& Brand, R. (1998). Intensive lifestyle changes for reversal of coronary heart disease. Journal of the American Medical Association, 280(23), 2001-2007. https://doi.org/10.1001/jama.280.23.2001

Palta, P., Page, G., Piferi, R. L, Gill, J. M., Hayat, M. J., Connolly, A. B., \& Szanton, S. L. (2012). Evaluation of a mindfulness-based intervention program to decrease blood pressure in low-income African American older adults. Journal of Urban Health, 89, 308-316. https://doi.org/10.1007/s11524-0119654-6

Purser, R. (2019). McMindfulness: How mindfulness became the new capitalist spirituality. Repeater Books.

Rees, B. (2011). Overview of outcome data of potential meditation training for soldier resilience. Military Medicine, 176(11), 1232-1242.

Schiraldi, G. R. (2009). The post-traumatic stress disorder sourcebook: A guide to healing, recovery, and growth (2nd ed.). McGraw-Hill.

Sheppard, S., Malatra, J., \& Israel, A. (2010). The impact of deployment on U.S. military families. American Psychologist. 65(6), 599-609. https://doi.org/10.1037/a0020332

Stankovic, L. (2011). Transforming trauma: A qualitative feasibility study of integrative restoration (iRest) yoga Nidra on combat-related posttraumatic stress disorder. International Journal of Yoga Therapy, $21,23-37$.

Stanley, E., Schaldach, J., Kiyonaga, A., \& Jha, A. (2011). Mindfulness-based mind fitness training: A case study of a high-stress pre-deployment military cohort. Cognitive and Behavioral Practice, 18(4), $566-576$.

Tick, E. (2005). War and the soul: Healing our nation's veterans from post-traumatic stress disorder. Quest Books.

Turner, S., Beidel, D., \& Freuh, C. (2005). Multicomponent behavioral treatment for chronic combat-related posttraumatic stress disorder. Behavior Modification, 29(1), 39-69. https://doi.org/10.1177/0145445504270872

U.S. Department of Veterans Affairs. (2017a). American's wars. https://www.va.gov/opa/publications/factsheets/fs americas wars.pdf

U.S. Department of Veterans Affairs. (2017b). VA/DOD clinical practice guideline for the management of posttraumatic stress disorder and acute stress disorder. https://www.healthquality.va.gov/guidelines/MH/ptsd/VADoDPTSDCPGFinalo12418.pdf

U.S. Department of Veterans Affairs. (2019a). Statistical trends: Veterans with a service-connected disability, 1990 to 2018. https://www.va.gov/vetdata/docs/Quickfacts/SCD trends_FINAL_2018.pdf

U.S. Department of Veterans Affairs. (2019b). Whole health for whole life. https://www.va.gov/patientcenteredcare/index.asp 
Veterans Affairs Healthcare Analysis and Information Group. (2011). 2011 Complementary and alternative medicine. Department of Veterans Affairs.

Vujanovic, A., Niles, B., Pietrefesa, A., Schmertz, S., \& Potter, C. (2011). Mindfulness in the treatment of posttraumatic stress disorder among military veterans. Professional Psychology: Research and Practice, 42(1), 24-31.

Weiler, L. (2013). Yoga teacher training. Human Kinetics.

Welcome Home Vets, Inc. (2011). Practical approaches to military population and self-care for therapists. Presented at the Loosen Your Grip Providers Conference, Oveido, FL.

Wong, A. (2018). Why schools are banning yoga. The Atlantic. https://www.theatlantic.com/education/archive/2018/o9/why-schools-are-banning-yoga/570904/

Zeiden, F., Martucci, K., Kraft, R., Gordon, N., McHaffie, J., \& Coghill, R. (2011). Brain mechanisms supporting the modulation of pain by mindfulness meditation. The Journal of Neuroscience, 31, $5540-5548$.

The Journal of Social, Behavioral, and Health Sciences (JSBHS), co-sponsored by the College of Health Sciences and the College of Social and Behavioral Sciences at Walden University, is a peer-reviewed, online, interdisciplinary journal focusing on theoretically-based research that addresses contemporary national and international issues. $J S B H S$ articles include peer-reviewed research reports, brief resports, comprehensive literature reviews, books reviews, and student research. 